\title{
Superluminal tunnelling through successive barriers: Does QM predict infinite group-velocities?
}

\author{
ERASMO RECAMI \\ Facoltà di Ingegneria, Università statale di Bergamo, 24044-Dalmine \\ (BG), Italy and I.N.F.N.-Sezione di Milano, Milan, Italy
}

(Received 22 November 2003)

\begin{abstract}
The phenomenon of one-dimensional non-resonant tunnelling is analyzed through two or more successive (opaque) potential barriers, separated by intermediate free regions $\mathcal{R}$, just by exploiting the relevant solutions to the Schroedinger equation. The total traversal time has been shown by us to be independent not only of the barrier widths (the so-called 'Hartman effect'), but also of the $\mathcal{R}$-widths: so that the effective group velocity in the regions $\mathcal{R}$, between two successive barriers, can be regarded as practically infinite. Such a prediction has been theoretically confirmed and generalized (as well as interpreted in terms of 'super-oscillations') by Aharonov et al. A recent experiment by Longhi et al. supported the predictions by considering two successive gratings in an optical fibre, that is, by having recourse to two 'classical barriers' (which allow simulating the tunnelling, due to the known formal identity between the Schrödinger and the Helmholtz equation).
\end{abstract}

\section{Introduction}

It is known within quantum mechanics, with regard to the tunnelling processes, that the tunnelling time - either evaluated as a simple 'phase time' [1] or calculated through analysis of the wavepacket behaviour [2] - does not depend on the barrier width in the case of opaque barriers. Such a phenomenon, sometimes called 'Hartman effect' [3], implies Superluminal and arbitrarily large (group) velocities $v$ inside long enough barriers [2]. Experiments that may verify this prediction by, say, electrons are difficult. Luckily enough, however, the Schrödinger equation in the presence of a potential barrier is mathematically identical [4] to the Helmholtz equation for an electromagnetic wave propagating, for instance, down a metallic waveguide along the $x$-axis [5-12]; and a barrier height $V$ bigger than the electron energy $E$ corresponds (for a given wave frequency) to a waveguide transverse size smaller than a cut-off value. A segment of undersized guide does therefore behave as a barrier for the wave (photonic barrier): the wave assumes therein — like an electron inside a quantum barrier — an imaginary momentum or wave number and gets exponentially damped along $x$, as a consequence. In other words, it becomes an evanescent wave (going back to normal propagation, even if with reduced amplitude, when the narrowing ends and the guide returns to its initial transverse size). Thus, a tunnelling experiment can be simulated by having recourse to evanescent waves (for which the concept of group velocity can be properly extended [5]). 


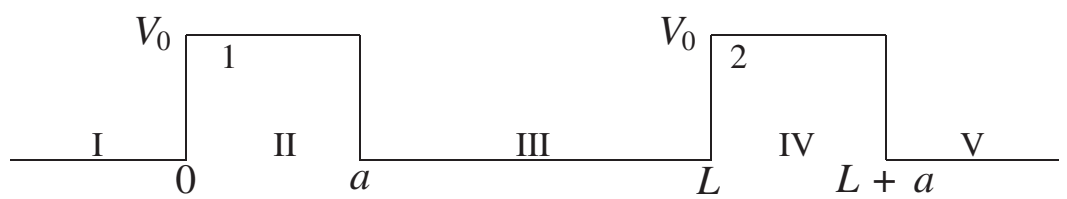

Figure 1. The non-resonant tunnelling process, through two successive (opaque) potential barriers, considered in this paper. We show that, far from resonances, the (total) phase time for tunnelling through the two barriers does not depend both on the barrier widths and on the distance between the barriers.

And the fact that evanescent waves travel with superluminal speeds has been actually verified in a series of famous experiments. Various experiments performed from 1992 onwards by R. Chiao's and A. Steinberg's group at Berkeley [6], by G. Nimtz et al. at Cologne [7], by A. Ranfagni and colleagues at Florence [7], and by others at Vienna, Orsay, Rennes [7] — verified that 'tunnelling photons' travel with superluminal group velocities; in other words, they confirmed, directly or indirectly, the occurrence of the Hartman effect.

Let us emphasize that the most interesting experimental setup, dealing with evanescent waves, seems to be, however, the one comprehending two successive evanescence regions ('classical barriers'), separated by a segment of normal region. For suitable frequency bands, i.e. far from resonances, it was found that the total crossing time does not depend on the length of the intermediate (normal) region: namely, that the beam speed along it is infinite. The related experimental results [8] have been confirmed by numerical simulations based on Maxwell equations only [9]. But they are so amazing that we want to check whether they agree also with what is predicted by quantum mechanics in the analogous case of two successive potential barriers.

In this note we show that, for non-resonant tunnelling through two successive, rectangular (opaque) potential barriers (figure 1), the (total) phase time does depend neither on the barrier widths nor on the distance between the barriers. In other words, far from resonances the tunnelling phase time, which does depend on the entering energy, can be shown to be independent of the distance between the two barriers.

\section{Phase time evaluation}

Let us consider the (quantum-mechanical) stationary solution for the onedimensional (1D) tunnelling of a non-relativistic particle, with mass $m$ and kinetic energy $E=\hbar^{2} k^{2} / 2 m=m v^{2} / 2$, through two equal rectangular barriers with height $V_{0}\left(V_{0}>E\right)$ and width $a$, quantity $L-a \geq 0$ being the distance between them. The Schrödinger equation is

$$
-\frac{\hbar^{2}}{2 m} \frac{\partial^{2}}{\partial x^{2}} \psi(x)+V(x) \psi(x)=E \psi(x)
$$

where $V(x)$ is zero outside the barriers, while $V(x)=V_{0}$ inside the potential barriers. In the various regions I $(x \leq 0)$, II $(0 \leq x \leq a), \quad$ III $(a \leq x \leq L)$, 
$\mathrm{IV}(L \leq x \leq L+a)$ and $\mathrm{V}(x \geq L+a)$, the stationary solutions to equation (1) are the following

$$
\left\{\begin{array}{l}
\psi_{\mathrm{I}}=\mathrm{e}^{+i k x}+A_{1 \mathrm{R}} \mathrm{e}^{-i k x} \\
\psi_{\mathrm{II}}=\alpha_{1} \mathrm{e}^{-\chi x}+\beta_{1} \mathrm{e}^{+\chi x} \\
\psi_{\mathrm{III}}=A_{1 \mathrm{~T}}\left[\mathrm{e}^{i k x}+A_{2 \mathrm{R}} \mathrm{e}^{-i k x}\right] \\
\psi_{\mathrm{IV}}=A_{1 \mathrm{~T}}\left[\alpha_{2} \mathrm{e}^{-\chi(x-L)}+\beta_{2} \mathrm{e}^{+\chi(x-L)}\right] \\
\psi_{\mathrm{V}}=A_{1 \mathrm{~T}} A_{2 \mathrm{~T}} \mathrm{e}^{i k x}
\end{array}\right.
$$

where $\chi \equiv \sqrt{2 m\left(V_{0}-E\right)} / \hbar$, and quantities $A_{1 \mathrm{R}}, A_{2 \mathrm{R}}, A_{1 \mathrm{~T}}, A_{2 \mathrm{~T}}, \alpha_{1}, \alpha_{2}, \beta_{1}$ and $\beta_{2}$ are the reflection amplitudes, the transmission amplitudes, and the coefficients of the 'evanescent' (decreasing) and 'anti-evanescent' (increasing) waves for barriers 1 and 2, respectively. Such quantities can be easily obtained from the matching (continuity) conditions:

$$
\begin{aligned}
& \left\{\begin{array}{l}
\psi_{\mathrm{I}}(0)=\psi_{\mathrm{II}}(0) \\
\left.\frac{\partial \psi_{\mathrm{I}}}{\partial x}\right|_{x=0}=\left.\frac{\partial \psi_{\mathrm{II}}}{\partial x}\right|_{x=0}
\end{array}\right. \\
& \left\{\begin{array}{l}
\psi_{\mathrm{II}}(a)=\psi_{\mathrm{III}}(a) \\
\left.\frac{\partial \psi_{\mathrm{II}}}{\partial x}\right|_{x=a}=\left.\frac{\partial \psi_{\mathrm{III}}}{\partial x}\right|_{x=a}
\end{array}\right. \\
& \left\{\begin{array}{l}
\psi_{\mathrm{III}}(L)=\psi_{\mathrm{IV}}(L) \\
\left.\frac{\partial \psi_{\mathrm{III}}}{\partial x}\right|_{x=L}=\left.\frac{\partial \psi_{\mathrm{IV}}}{\partial x}\right|_{x=L}
\end{array}\right. \\
& \left\{\begin{array}{l}
\psi_{\mathrm{IV}}(L+a)=\psi_{\mathrm{V}}(L+a) \\
\left.\frac{\partial \psi_{\mathrm{IV}}}{\partial x}\right|_{x=L+a}=\left.\frac{\partial \psi_{\mathrm{V}}}{\partial x}\right|_{x=L+a}
\end{array}\right.
\end{aligned}
$$

Equations (3)-(6) form eight equations for the eight unknowns $\left(A_{1 \mathrm{R}}, A_{2 \mathrm{R}}, A_{1 \mathrm{~T}}\right.$, $A_{2 \mathrm{~T}}, \alpha_{1}, \alpha_{2}, \beta_{1}$ and $\beta_{2}$ ). First, let us obtain the four unknowns $A_{2 \mathrm{R}}, A_{2 \mathrm{~T}}, \alpha_{2}, \beta_{2}$ from equations (5) and (6) in the case of opaque barriers, i.e. when $a$ is large enough (and $\chi$ not too small) so that one can assume that $\chi a \rightarrow \infty$ :

$$
\left\{\begin{array}{l}
\alpha_{2} \longrightarrow \mathrm{e}^{i k L} \frac{2 i k}{i k-\chi} \\
\beta_{2} \longrightarrow \mathrm{e}^{i k L-2 \chi a} \frac{-2 i k(i k+\chi)}{(i k-\chi)^{2}} \\
A_{2 \mathrm{R}} \longrightarrow \mathrm{e}^{2 i k L} \frac{i k+\chi}{i k-\chi} \\
A_{2 \mathrm{~T}} \longrightarrow \mathrm{e}^{-\chi a} \mathrm{e}^{-i k a} \frac{-4 i k \chi}{(i k-\chi)^{2}}
\end{array}\right.
$$


Then, we may obtain the other four unknowns $A_{1 \mathrm{R}}, A_{1 \mathrm{~T}}, \alpha_{1}, \beta_{1}$ from equations (3) and (4). Again in the case of large enough barriers (and $\chi a \rightarrow \infty$ ), one gets:

$$
\left\{\begin{array}{l}
\alpha_{1} \longrightarrow \frac{2 i k}{i k-\chi} \\
\beta_{1} \longrightarrow \mathrm{e}^{-2 \chi a}(k-i \chi) \frac{\sin k(L-a)}{\chi} A \\
A_{1 \mathrm{R}} \longrightarrow \frac{i k+\chi}{i k-\chi} \\
A_{1 \mathrm{~T}} \longrightarrow \mathrm{e}^{-\chi a} \mathrm{e}^{-i k L} A,
\end{array}\right.
$$

where

$$
A \equiv \frac{2 \chi k}{2 \chi k \cos k(L-a)+\left(\chi^{2}-k^{2}\right) \sin k(L-a)}
$$

results, incidentally, to be real.

At this point, by applying the well-known definition of phase-time (see, for instance, [1-3]), we can derive that the tunnelling time

$$
\begin{aligned}
\tau_{\text {tun }}^{\mathrm{ph}} \equiv \hbar \frac{\partial \arg \left[A_{1 \mathrm{~T}} A_{2 \mathrm{~T}} \mathrm{e}^{i k(L+a)}\right]}{\partial E} & =\hbar \frac{\partial}{\partial E} \arg \left[\frac{-4 i k \chi}{(i k-\chi)^{2}}\right] \\
& =\hbar \frac{\partial}{\partial E} \arctan \left[\frac{k^{2}-\chi^{2}}{k \chi}\right]=\frac{1}{\hbar \chi} \frac{2 m}{k},
\end{aligned}
$$

while depending on the energy of the tunnelling particle, does not depend on $L+a$ (it being actually independent both of $a$ and $L$ ).

This result does not only confirm the so-called 'Hartman effect' [2, 3, 13-16] for the two opaque barriers - i.e. the independence of the tunnelling time from the opaque barrier widths - but it also extends such an effect by implying the total tunnelling time to be independent of $L$ (cf. figure 1). Equation (10) shows the considered tunnelling time to represent solely the time spent inside the first barrier, without any contribution coming in particular from the intermediate region. It can be regarded as further evidence of the fact that quantum systems seem to behave as non-local [17-22], but it has a more general meaning, it being associated with the properties of any waves (and, in fact, something very similar happens also with electromagnetic waves: see below). It is important to stress once more that the previous result holds, however, for non-resonant (nr) tunnelling: i.e. for energies far from the resonances that arise in region III due to interference between forward and backward travelling waves (a phenomemon analogous to the FabryPérot (or Bragg) waves in the case of classical waves). Otherwise, it is known that the expression for the time delay $\tau$ near a resonance is rather larger: for example, for a Gaussian resonance at $E_{\mathrm{r}}$ with half-width $\Gamma$, it would be $\tau=\hbar \Gamma\left[\left(E-E_{\mathrm{r}}\right)^{2}+\Gamma^{2}\right]^{-1}+\tau_{\mathrm{nr}}$.

It may be interesting to recall that tunnelling durations have been analysed also within relativistic quantum mechanics, and precisely in connection with the Dirac equation [23]; and that a phenomenon like the Hartman's effect has been found also in that case.

Let us add one more comment. From some papers [24], it seems that the penetration time needed to cross a portion of a barrier in the case of a very long barrier starts to increase again — after the plateau corresponding to infinite 
speed - proportionally to the distance. This seems to be due to the contribution of the above-barrier frequencies (or energies) contained in the considered wave packets, which become more and more important as the tunnelling components are progressively damped down. In this paper, however, we refer to the behaviour of the tunnelling waves (or, in the classical case, of the evanescent waves) only.

Some real deviations from the $\mathrm{HE}$ are going to be discussed in the Appendix.

\section{Discussion}

The tunnelling-time independence from the width $a$ of each one of the two opaque barriers is itself a generalization of the Hartman effect; and may, in general, be described — following $[4,6]$ (see also $[25,26]$ ) — on the basis of the reshaping phenomenon which takes place inside each barrier. But we may reserve the term 'Generalized Hartman effect' to the phase-time independence from $R$.

Also this interesting tunnelling-time independence from the distance $R \equiv L-a$ between the two barriers can be described on the basis of the interference between the waves coming from the first barrier (region II) and travelling in region III and the waves reflected from the second barrier (region IV) back into the same region III. This interference may cause [2, 13-17, 20, 27] an 'advance' (i.e. an effective acceleration) of the incoming waves.

Such a phenomenon would be similar to the analogous advance expected even in region I; indeed, going on to the wave packet language, let us recall that we have noticed in $[2,13,16,20,27]$ that the arriving wave packet interferes with the reflected waves that start to be generated as soon as the packet forward tail reaches the (first) barrier edge: in such a way that (already before the barrier) the backward tail of the initial wavepacket decreases - for destructive interference with those reflected waves - at a larger degree than the forward one. This simulates an increase of the average speed of the entering packet: hence, the effective (average) flight-time of the approaching packet from the source to the barrier does decrease.

So, as we were saying, reshapings and 'advancements' of the same kind (inside the barriers, as well as to the left of the barriers) might be a priori able to describe how the tunnelling-time is independent of the barrier widths and of the distance between the two barriers. In any case, phenomena of this kind do not seem to be at variance with Special Relativity, as has been discussed in a number of papers (cf. [9] and [5], and references therein). It remains impressive, nevertheless, that in region III - where no potential barrier is present, the current is non-zero and the wavefunction is oscillatory - the effective speed (or group velocity) is practically infinite. (Loosely speaking, one might say that the considered multi-barrier setups can behave in some cases as 'space destroyers' with reference to their intermediate regions...). After some straightforward but rather bulky calculations, one can moreover see that the same effects (i.e., the independence from the barrier widths and from the distances between the barriers) are still valid for any number of barriers, with different widths and different distances between them: as it has been explicitly shown by Esposito [28].

Our previous predictions [28] have been theoretically confirmed and generalized (and explained in terms of 'superoscillations') by Aharonov et al. in [29]. Indeed, those authors have found that, according to QM, a wave packet can travel, 
in zero time and with negligible distortion, a distance arbitrarily larger than the width of the wave packet.

From the experimental point of view our predictions have received further experimental confirmation in [30], where two gratings (figure 2) were used in an optical fibre, as suggested in [28] as well as above. Let us recall once more that the similarity between photon and (non-relativistic) particle tunnelling [2, 4, 10, 11, $13,16,17,27,33-36$; see also 18, 26] implies that these results hold also for photon tunnelling through successive 'barriers': for example, for photons in the presence of two successive band-gap filters.

When investigating the tunnelling of optical pulses through double-barrier periodic fibre Bragg gratings, measurement of the tunnelling time has confirmed that, far from the (Bragg) resonances of the apparatus, the transit time is practically independent of the distance between the barriers: attention is called, in particular, to the last figure of [30], which is figure 3 of the present paper. Further experiments should easily be realizable, by having recourse to two or more photonic crystals; indirect experimental evidence has already come from papers [18, 32, 37]. For further details, one may check recent work by Longhi et al. [31, 32].

No less interesting would be experiments performed actually within the quantum realm, whenever a succession of two or more potential barriers are present.

Let us repeat, in this discussion, that the classical, relativistic (stationary) Helmholtz equation for an electromagnetic wave packet is formally identical to the quantum, non-relativistic (stationary) Schrödinger equation. ${ }^{@}$ Thus all our results have to be valid also for electromagnetic wave propagation along metallic waveguides, or optical fibres, with a succession of classical barriers: these can be a

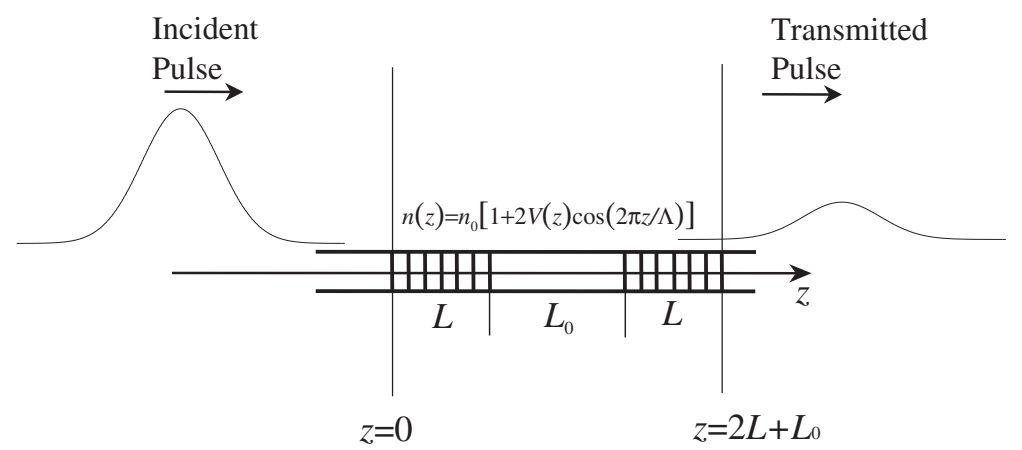

Figure 2. Scheme of the apparatus adopted in [30] for checking our theoretical predictions. As (classical) barriers, there have been used two gratings, with the same width, in an optical fibre. Notice that in [30] there were chosen symbols different from ours (e.g., quantity called $L$ in our figure 1 is called $L_{0}$ in this figure, taken from [30]).

${ }^{(a)}$ Even if these equations are different (due to the different order of the time derivative) in the time-dependent case. Indeed, we have shown, in section 10 of the third reference in [2], that they still have in common classes of analogous solutions, differing only in their spreading properties [2, 11, 38, 39; and also 13, 16]. 


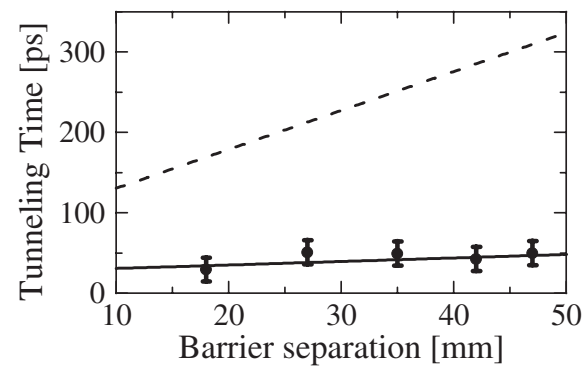

Figure 3. Results of the experiment [30] whose set-up is represented in figure 2. The tunnelling time spent by the considered pulse for traveling from $z=0$ to $z=2 L+L_{0}$ (i.e., from the initial edge of the first barrier to the final edge of the second barrier) does not depend on the distance $L_{0}$ between the two barriers. In the intermediate region, therefore, the pulse group-velocity appears to be practically infinite.

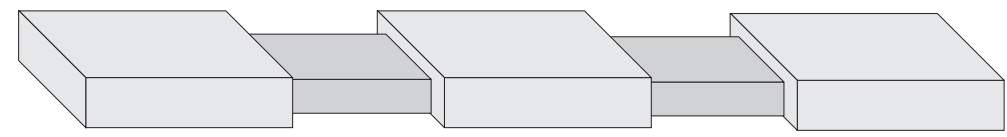

Figure 4. Two classical barriers can be constituted also by two segments of undersized waveguide, i.e., segments with a transverse size smaller than a cut-off value (depending on the frequency of the wave which travels along the waveguide). This is the set-up used in the pioneering experiment described in [8].

succession of undersized segments in a metallic waveguide (figure 4), or of gratings in an optical fiber (figure 2), and so on. As a matter of fact, the tunnelling of a particle under and along a potential barrier was successfully simulated $[2,4,7-11$, $13,16,21,33,34,40-44]$ by the travelling of evanescent waves along an undersized metallic waveguide. This agrees with the numerical calculations performed (in connection with the former case) directly from Maxwell equations [9, 13, 18], besides having been verified by the known series of early 'tunnelling' experiments with microwaves (cf. [7, 26, 36, 41-43, 45] and particularly [8, 30, 46]).

\section{Acknowledgements}

The author is very grateful to the organizers of the present international workshop, and particularly to R.Bonifacio, for very stimulating discussions and kind invitation. This paper is based on work performed in collaboration with V. S. Olkhovsky and G. Salesi. Thanks are due also to G. Bonera, S. Esposito, M. Villa, as well as to F. Bassani, C. Becchi, G. Benedek, L. Bosi, G. C. Cavalleri, R. Y. Chiao, G. C. Costa, G. Degli Antoni, F. Fontana, R. Garavaglia, A. Gigli Berzolari, H. E. Hernández-Figueroa, J. Jakiel, E. Kapuschik, G. Kurizki, G. Marchesini, R. Mignani, D. Mugnai, M. Palis, M. Pernici, A. Ranfagni, A. Shaarawi, A. Steinberg and M. Zamboni-Rached for scientific collaboration and kind interest. At last, for their active cooperation, special thanks are due to $G$. Giuffrida, M. T. Vasconselos and particularly to G. La Pietra. 


\section{Appendix: Negative speeds and time advances}

We discuss in this Appendix some interesting deviations from the Hartman Effect (HE). We refer here to the case of one potential barrier only; and follow the notation introduced in [2].

In [47] it was noticed (see also [48, 49]) that, for initial Gaussian wave packets $\Psi(x, t)$ with non-real weight amplitudes $G(k-\bar{k})$, when $\left.\left\langle t\left(x_{i}\right)\right)\right\rangle_{\text {in }}=0$ for $x_{i}<0$ with $\left|x_{i}\right| \gg a$, the values $\left\langle\tau_{\text {tun }}\right\rangle$ decrease for $\Delta k>\sim 0.02 \mathrm{~A}^{-1}$, and can even become negative for $\Delta k>\sim 0.05 \mathrm{~A}^{-1}$. More precisely, in the referenced papers, and in particular in [49], the important fact has been shown theoretically that, with increasing $a$, the quantity $\left\langle\tau_{\text {tun }}\right\rangle$, after reaching a value equal to (or, sometimes, even smaller than) the HE plateau value, starts diminishing and the effective tunnelling velocity does increase correspondingly, even to a larger extent than predicted by the HE. At a certain point, the total tunnelling time $\left\langle\tau_{\text {tun }}\right\rangle$ can become negative; so that, in the case of bumped wave packets, the outgoing (tunnelled) peak leaves the barrier before the incoming peak entered it.

As we are going to mention below, and as was predicted elsewhere [21, 22, 50], such remarkable phenomena are associated with the intervention of 'negative speeds' [22, 31, 50]. Again, a possible description of such advancements may be found in terms of the very rapid spreading of the initial and transmitted wave packets for large $\Delta k$ values.

In connection with the known similarities between tunnelling (quantum) packets and evanescent (classical) beams, it can be noticed that all the wave equations, as well as Helmholtz's, are relativistic-like, even when one fixes his attention on waves different from electromagnetic ones. It is therefore in order to see what it has been possible to predict, with respect to the 'negative speeds', from the more general point of view of Special Relativity. To this purpose, let us here recall that: (i) even if all the ordinary causal paradoxes, invented for tachyons, seem to be solvable within Special Relativity, when it is not restricted to subluminal motions only [40, 50]; (ii) nevertheless, whenever we have an object $\mathcal{O}$ travelling at superluminal speed, negative contributions ought then to be expected to the tunnelling times [22]: and this should not to be regarded as unphysical [50]. In fact, whenever the object $\mathcal{O}$ overtakes the infinite speed with respect to a certain observer, it will afterwards appear to the same observer as its anti-object $\overline{\mathcal{O}}$ travelling in the opposite space direction [50]. For instance, when going from the lab to a frame $\mathcal{F}$ moving in the same direction — with whatever (even very small) speed - as the particles or waves enter the barrier region, the objects $\mathcal{O}$ penetrating the final part of the barrier (with almost infinite speed [21]) will appear in the frame $\mathcal{F}$ as anti-objects $\overline{\mathcal{O}}$ crossing that portion of the barrier in the opposite space-direction [50]. In the new frame $\mathcal{F}$, such anti-objects $\overline{\mathcal{O}}$ would yield a negative contribution to the tunnelling time: which could even result, in total, to be negative. What we want to stress here is that the appearance of such negative times is predicted by Relativity itself, on the basis of the ordinary postulates [21, 22, 50]. For a theoretical point of view, besides [21, 22, 50], see [31]. For a (quite interesting!) experimental point of view, see [32].

Negative times seem to have been revealed even near nuclear resonances, distorted by the non-resonant background (see, in particular, [51]). 


\section{References}

[1] Hauge, E. H., and Støvneng, J. A., 1989, Rev. Mod. Phys., 61, 917.

[2] Olkhovsky, V. S., and Recami, E., 1992, Phys. Rep., 214, 340; Olkhovsky, V. S., Recami, E., Raciti, F., and Zaichenko, A. K., 1995, J. Phys.-I (France), 5, 1351; Olkhovsky, V. S., Recami, E., and Jakiel, J., "A new time-analysis of photon and (non-relativistic) particle tunnelling", quant-ph/0102007, to appear in Phys. Reports.

[3] Hartman, T. E., 1962, J. Appl. Phys., 33, 3427.

[4] Chiao, R. Y., Kwiat, P. G., and Steinberg, A. M., 1991, Physica, B175, 257; Martin, Th., and Landauer, R., 1992, Phys. Rev., A45, 2611; Ranfagni, A., Mugnai, D., Fabeni, P., and Pazzi, G. P., 1991, Appl. Phys. Lett., 58, 774.

[5] Recami, E., Fontana, F., and Garavaglia, R., 2000, Int. J. Mod. Phys., A15, 2793.

[6] Steinberg, A. M., Kwiat, P. G., and Chiao, R. Y., 1993, Phys. Rev. Lett., 71, 708; Chiao, R. Y., Kwiat, P. G., and Steinberg, A. M., 1993, Sci. Am., 269(12), 38.

[7] Enders, A., and Nimtz, G. J. 1993, Phys.-I (France), 3, 1089; Ranfagni, A., Fabeni, P., Pazzi, G. P., and Mugnai, D., 1993, Phys. Rev., E48, 1453; Spielmann, Ch., Szipocs, R., Stingl, A., and Krausz, F., 1994, Phys. Rev. Lett., 73, 2308; Balcou, Ph., and Dutriaux, L., 1997, Phys. Rev. Lett. 78, 851; Laude, V., and Tournois, P., J. 1999, Opt. Soc. Am., B16, 194.

[8] Nimtz, G., Enders, A., and Spieker, H., 1993, Wave and Particle in Light and Matter, edited by A. van der Merwe and A. Garuccio (New York: Plenum); 1994, J. de Physique-I, 4, 565; Enders, A., and Nimtz, G., 1993, Phys. Rev., B47, 9605.

[9] Barbero, A. P. L., Hernández H. E. F., and Recami, E., 2000, Phys. Rev., E62, 8628; Brodowsky, H. M., Heitmann, W., and Nimtz, G., 1996, Phys. Lett., A222, 125.

[10] Japha, Y., and Kurizki, G., 1996, Phys. Rev., A53, 586; Causality and interference in electron and photon tunnelling, preprint, Weizmann Inst. Sc., Rehovot, Jan. 1996; Causality and non-locality in 'faster-than-light' photon travel, preprint, Weizmann Inst. Sc., Rehovot, Sept. 1996.

[11] Olkhovsky, V. S., and Agresti, A., Tunneling and its Applications (Singapore: World Scientific, 1997), pp. 327-355; Jakiel, J., Olkhovsky, V. S., and Recami, E., 1998, Phys. Lett., A248, 156; Shatrawi, A. M., and Besieris, I. M., 2000, J. Phys. A: Math. Gen., 33, 8599, 7255, 7227; 2000, Phys. Rev., E62, 7415.

[12] Recami, E., 2001, Found. Phys., 31, 1119.

[13] Olkhovsky, V. S., and Recami, E., 1992, Phys. Rep., 214, 339.

[14] Bosanac, S., 1983, Phys. Rev., A28, 577.

[15] Fletcher, J. R., 1985, J. Phys., C18, L55.

[16] Olkhovsky, V. S., Recami, E., and Zaichenko, A. K., 1994, Solid State Commun., 89, 31.

[17] Olkhovsky, V. S., Recami, E., and Salesi, G., Tunneling through two successive barriers and the Hartman (Superluminal) effect, e-print quant-ph/0002022.

[18] Zamboni-Rached, M., Recami, E., and Fontana, F., 2001, Phys. Rev., E64, no.066603; Zamboni-Rached, M., Fontana, F., and Recami, E., 2003, Phys. Rev. E67, 036620; Zamboni-Rached, M., Nóbrega, K. Z., Recami, E., and Hernández, H. E. F., 2002, Phys. Rev., E66, 046617; Recami, E., 1998, Physica, A252, 586, and refs. therein.

[19] Chu, S., and Wong, S., 1982, Phys. Rev. Lett., 48, 738. See also [32].

[20] Mugnai, D., Ranfagni, A., Ruggeri, R., Agresti, A., and Recami, E., 1995, Phys. Lett., A209, 227.

[21] Barbero, A. P. L., Hernández, H. E., and Recami, E., 2000, Phys. Rev., E62, 8628; Recami, E., Hernández, H. E. F., and Barbero, A. P. L., 1998, Ann. Phys. (Leipzig), 7, 764. See also [5, 13, 27].

[22] See [2, p.1361]. See also [50].

[23] Petrillo, V., and Refaldi, L., 2003, Phys. Rev., A67, 012110; Krekora, P., Su, Q., and Grobe, R., 2001, Phys. Rev., A63, 032107; 2001, A64, 022105; Leavens, C. R., and Sala-Mayato, R., 1998, Ann. Phys. (NY), 7, 662.

[24] Abolhasani, M., and Golshani, M., 2000, Phys. Rev., A62; Leòn, J., (private communication); NimTz, G., (private communication). See also [49]. 
[25] Martin, Th., and Landauer, R., 1992, Phys. Rev., A45, 2611; Tsai, T., and Thomas, G., 1976, Am. J. Phys. 44, 636; Hupert, J. J., 1977, Am. J. Phys. 45, 210, and refs. therein.

[26] Ranfagni, A., Mugnai, D., Fabeni, P., and Pazzi, G. P., [4]; Ranfagni, A., Mugnai, D., and Agresti, A., 1993, Phys. Lett., A175, 334.

[27] Olkhovsky, V. S., Recami, E., Raciti, F., and Zaichenko, A. K., [2]; Abolhasani, M., and Golshani, M., [24]. See also Petrillo, V., [31].

[28] Olkhovsky, V. S., Recami, E., and Salesi, G., 2002, Europhys. Lett., 57, 879-884. Esposito, S., 2003, Phys. Rev., E67, 016609.

[29] Aharonov, Y., Erez, N., and Reznik, B., 2002, Phys. Rev., A65, 052124.

[30] Longhi, S., Laporta, P., Belmonte, M., and Recami, E., 2002, Phys. Rev., E65, 046610 .

[31] Garret, C. G. B., and Cumber, M. D. E., 1970, Phys. Rev., A1, 305; Chiao, R. Y., Kozhekin, A. E., and Kurizki, G., 1996, Phys. Rev. Lett., 77, 1254; Shaarawi, A. M., Tawfik, B. H., and Besieris, I. M., 2002, Phys. Rev., E66, 046626. See also Longhi, S., 2001, Phys. Rev., E64, 037601; Leavens, C. R., and Sala-Mayato, R., 1998, Ann. Phys. (Leipzig), 7, 662; Muga, M. G., and Palao, J. P., 1998, Ann. Phys. (Leipzig), 7, 671; Muga, J. G., Egusquiza, I. L., Damborenea, J. A., and Delgado, F., 2002, Phys. Rev., A66, 042115; Porras, M. A., Gonzalo, I., and Mondello, A., physics/0202069; Petrillo, V., and Refaldi, L., A time asymptotic expression for the wave function emerging from a quanto-mechanical barrier, subm. for pub., 2002; as well as the classical review-paper by G. Toraldo di Francia, 1952, Supplem. Nuovo Cim., 9(3), 391.

[32] Chu, S., and Wong, W., 1982, Phys. Rev. Lett., 48, 738; Segard, B., and Macke, B., 1985, Phys. Lett., A109, 213; Mitchell, M. W., and Chiao, R. Y., 1997, Phys. Lett., A230, 133; Nimtz, G., Eur. Phys. J.B (to appear as a Rapid Note); Wang, L. J., Kuzmich, A., and Dogariu, A., 2000, Nature, 406, 277. See also Longhi, S., Marano, M., Laporta, P., Belmonte, M., and Crespi, P., 2002, Phys. Rev., E65, 045602(R).

[33] Jakiel, J., Olkhovsky, V. S., and Recami, E., [11].

[34] Chino, R. Y., Kwiat, P. G., and Steinberg, A. M., 1991, Physica, B175, 257.

[35] Ranfagni, A., Mugnai, D., Fabeni, P., and Pazzi, G., 1991, Appl. Phys. Lett., 58, 774; Mugnai, D., Ranfagni, A., Ruggeri, R., and Agresti, A., 1992, Phys. Rev. Lett., 68, 259; 1993, Phys. Lett., A175, 334.

[36] Steinberg, A. M., Kwiat, P. G., and Chiao, R. Y., 1993, Phys. Rev. Lett., 71, 708; Chiao, R. Y., Kwiat, P. G., and Steinberg, A. M., 1993, Sci. Am., 269, 38.

[37] Garrett, C. G. B., and McCumber, D. E. M., 1970, Phys. Rev., A1, 305. See also $[19,32]$.

[38] Olkhovsky, V. S., 1998, Fizika Zhivogo (Physics of the Alive) 6, 17; in Mysteries, Puzzles and Paradoxes in Quantum Mechanics, edited by R. Bonifacio (Woodbury, NY:AIP, 1999), pp. 272-276.

[39] Olkhovsky, V. S., 1983, Ukrainskiy Fiz. Zhurnal, 28, 1319 [in Russian and Ukrainian].

[40] Zamboni-Rached, M., Recami, E., and Fontana, F., 2001, Phys. Rev., E64, 066603; cf. also Recami, E., Fontana, F., and Garavaglia, R., ref. [5].

[41] Ranfagni, A., Fabeni, P., Pazzi, G. P., and Mugnai, D., 1993, Phys. Rev., E48, 1453.

[42] Spielman, Ch., Szipoecs, R., Stingl, A., and Krausz, F., in [7]; Blaauboer, M., Kofman, A. G., Kozhekin, A. E., Kurizki, G., Lenstra, D., and Ladder, A., 1998, Phys. Rev., A57, 4905; Pereyra, P., 2000, Phys. Rev. Lett., 84, 1772; Longhi, S., Marano, M., Laporta, P., and Belmonte, M., 2001, Phys. Rev., E64, 055602(R).

[43] Balcou, Ph., and Dutriaux, L., in [7]; Laude, V., and Tournois, P., in [7]; Haibel, A., Nimtz, G., and Stahlhofen, A. A., 2001, Phys. Rev., E63, 047601. See also Zhu, S., Yu, A. W., Hawley, D., and Roy, R., 1986, Am. J. Phys., 54, 601.

[44] Brodowsky, H. M., Heitmann, W., and Nimtz, G., [9].

[45] Enders, A., and Nimtz, G., 1992, J. Phys.-I (France) 2, 1693; 1993, Phys. Rev., E48, 632; Nimtz, G., in Tunneling and its Applications (Singapore, 1997), pp. 223-237. 
An early experiment, however, had already been performed by CARniglia, C. K., and Mandel, L., 1971, J. Opt. Soc. Am., 61, 1035.

[46] Nimtz, G., Enders, A., and Spieker, H., in [8].

[47] Leavens, C. R., and Aers, G. C., 1989, Phys. Rev., B39, 1202.

[48] Hauge, E. H., Falck, J. P., and Fjeldly, T. A., 1987, Phys. Rev., B36, 4203.

[49] Petrillo, V., and Refaldi, L., 2000, Opt. Commun., 186, 35; Petrillo, V., and Refaldi, L., [23]. See also Refaldi, L., MSc thesis (Petrillo, V., Bonifacio, R., Recami, E., supervisors), Phys. Dept., Milan Univ., 2000.

[50] Recami, E., 1986, Rivista Nuovo Cim., 9(6), 1; Recami, E., Found. 1987, Phys., 17, 239; 1985, Lett. N. Cim., 44, 587.

[51] Olkhovsky, V. S., 1984, Sov. J. Part. Nucl., 15, 130; Nukleonika, 1990, 35, 99; Atti Accad. Pelor. Sci. Fis. Mat. Nat., 1992, 70, 21. 